www.jmscr.igmpublication.org Impact Factor 5.244

Index Copernicus Value: 83.27 ISSN (e)-2347-176x ISSN (p) 2455-0450 crossref DOI: _https://dx.doi.org/10.18535/jmscr/v4i11.59

\title{
Correlation of Modified Computed Tomography Severity Index with Complications of Acute Pancreatitis
}

\author{
Authors \\ Adhishwari Priyadarshini Parhi', S. Nisa ${ }^{2}$, B. B. Panda ${ }^{2}$, B. Dash ${ }^{2}$, S. Bhagat $^{3}$ \\ Jr. Resident ${ }^{1}$, Associate Professor ${ }^{2}$, Professor $^{3}$ \\ Department of Rediodiagnosis, VSS Institute of Medical Science and Research, Burla, Odisha, India \\ Email: adhiswaripriya@gmail.com
}

\begin{abstract}
Aims and Objectives:1.To identify the CT features of clinically suspected acute pancreatitis and grade the features according to modified CT severity index.2.to correlate the modified CT severity index with clinical outcome of patients.

Materials and Methods: Contrst enhanced CT scan was done in 50 patients referred from various clinical departments to the department of radio diagnosis, V S S Institute of Medical Science and Research, Burla. Study period was sept 2014 to sept 2016. CT features were scored according to modified CT severity index. Patients complications which were taken into consideration non the basis of duration of hospital stay, organ failure, surgical intervention, systemic infection and mortality.

Results and Conclusion: modified CT severity score was found to be significantly correlated with organ failure $(p<0.002)$, systemic infection $(p=0.001)$ and duration of hospital stay $(p<0.01)$. The inclusion of extra pancreatic parenchymal complication in the modified CT severity scoring makes it a better prognostic indicator than CT severity scoring, used earlier, for assessing adverse clinical outcomes in patients with acute pancreatitis.

Keywords: modified CT severity index, acute pancreatitis, complications.
\end{abstract}

\section{INTRODUCTION}

Acute pancreatitis is one of the common cause of acute abdomen leading to emergency visit in both developed and developing countries. Acute pancreatitis along with its complications is a significant cause of morbidity in hospitalised patients and known to run an unpredictable course. The 1992 international Atlanta Symposium definition of acute pancreatitis is the one which many pancreatologists use. According to it, pancreatitis is an acute inflammatory process of the pancreas with variable involvement of other regional tissues or remote organ systems. According to 2012 Revised Atlanta classification, Acute pancreatitis is best defined clinically by a patient presenting with any two of the following criteria: (1) Symptoms, such as epigastric pain, consistent with the disease; (2) A Serum amylase or lipase greater than three times the upper limit of normal; (3) Radiologic imaging consistent with the diagnosis, usually using computed tomogramphy (CT) or magnetic resonance imaging (MRI). Now-a-days incidence of acute pancreatitis is increasing due to change in lifestyle of population i.e. increase intake of alcohol and becoming overweight that leads to cholelithiasis. Treatment 
of patients with acute pancreatitis is based on the initial assessment of disease severity .

Acute Pancreatitis morphologically can be mild interstitial (edematous) or severe necrotizing pancreatitis. Severe necrotizing pancreatitis occurs in approximately $20 \%$ of patients and is associated with organ failure or local complications of necrosis ,infection or pseudocyst. Present study was conducted to establish the ability of CT in patients of clinically suspected acute pancreatitis referred from various clinical departments in depicting and quantifying pancreatic parenchymal injury by modified CT severity index and is used to evaluate the prognosis of the patients with acute pancreatitis .CT severity index was used initially. This scoring system is based on pancreatic morphology, number of peri-pancreatic fluid collections and pancreatic necrosis. Now Modified Computed Tomography Severity Index (MCTSI) has been introduced which differs from the Computed Tomography Severity Index (CTSI) by including the presence of extra pancreatic complications and grading the peripancreatic fluid collection in terms of presence or absence instead of the number of fluid collections.

\section{AIM AND OBJECTIVES}

1. To grade the CT findings of patients with acute pancreatitis according to modified CT severity index

2. to correlate the grade with patient outcome

Table-1: Modified CT severity index

\begin{tabular}{|c|c|}
\hline Prognostic Indicators & scores \\
\hline Pancreatic inflammation & \\
\hline *Normal pancreas & 0 \\
\hline *Intrinsic pancreatic & 2 \\
\hline abnormality with or without & \\
\hline $\begin{array}{l}\text { inflammatory changes in the } \\
\text { peripancreatic fat }\end{array}$ & 4 \\
\hline *Pancreatic or peripancreatic & \\
\hline $\begin{array}{l}\text { fluid collections or } \\
\text { peripancreatic fat stranding }\end{array}$ & 0 \\
\hline Pancreatic necrosis & 2 \\
\hline *None & 4 \\
\hline$* \leq 30 \%$ & \\
\hline$*>30 \%$ & \\
\hline $\begin{array}{l}\text { Extrapancreatic } \\
\text { complications }\end{array}$ & \\
\hline *(one or more of Ascites, & 2 \\
\hline
\end{tabular}

Pleural effusion,

Extrapancreatic parenchymal

complications, gastrointestinal involvement,

Vascular complications)

As shown in table-1 Modified Severity Index = CT grade + percentage necrosis + extrapancreatic complications (points) Mild : $(0-2)$

Moderate: $(4-6)$

Severe : $(8-10)$

\section{MATERIALSAND METHODS}

Source of data-cases of acute pancreatitis subjected to contrast enhanced CT scan in department of radiodiagnosis in V S S Institute of Medical Science and Research, Burla.

Study period -2 years (September 2014 September 2016)

Study design- Hospital based prospective study

Method of data collection-cases of acute pancreatitis graded according to modified CT severity index as shown in the table-1 and were assessed on 5th day of initial CT and on the day of discharge as 1.development of organ failure as respiratory failure (defined as $\mathrm{Po} 2<60 \mathrm{~mm} \mathrm{Hg}$ ), shock ( systolic BP of $<90 \mathrm{~mm} \mathrm{Hg}$ and tachycardia $>130$ ), renal failure ( serum creatinine $>2 \mathrm{mg} / \mathrm{dl}$ ), abdominal bleeding (>500 ml / $24 \mathrm{hr}$ ), central nervous system failure (Glasgow Coma Scale score of less than 6 in the absence of sedation or by the sudden onset of confusion or psychosis), hepatic failure (serum bilirubinlevels greater than 100 $\mu \mathrm{mol} / \mathrm{L}$ or alkaline phosphatase levels greater than three times theupper limit of the normal range) and hematologic system failure (hematocrit level of less than $20 \%$, WBC of less than $2,000 / \mathrm{mm} 3$, or platelet count of less than $40,000 / \mathrm{mm} 3.2$.length of hospital stay,3.infection 4.surgical intervention 5. mortality

INCLUSION CRITERIA-all patients of acute pancreatitis confirmed by serum amylase and lipase subjected to contrast enhanced CT scan.

EXCLUSION CRITERIA-(1) Patients with chronic pancreatitis. (2) Patients discharged from hospital before they recover from organ failure

\section{RESULTS}

The study was carried out involving total 50 patients diagnosed with acute pancreatitis by raised serum 
amylase and lipase. Study included patients between age group ranging from 15 to 79 years with mean age of 40 with maximum cluster of cases are seen in the range of $26-35$ that is $22(44 \%)$. There were 41 male patients constituting $82 \%$ and 9 female patients constituting $18 \%$ of the study group with a ratio of 4.5:1 indicating acute pancreatitis is more prevalent in males. This is due to more susceptibility of the male patients for the etiological factors causing acute pancreatitis. This is attributed mainly to alcoholism in the present study. In our study 28 (56\%) were alcoholic, 14(28\%) had cholelithiasis and the remaining patients were grouped as others which consisted of 8 patients , diagnosed as idiopathic $10 \%(n=5)$, trauma $4 \%(n=2)$, pancreatic carcinoma $2 \%(n=1)$. Out of 50 patients, all presented with pain abdomen (100\%), 28\% with vomiting, and $7 \%$ with fever.

According to figure $-120(40 \%)$ patients out of 50 showed mild (0-2) grade ,21 (42\%) showed moderate(4-6) and9(18\%) showed severe(8-10)type of acute pancreatitis

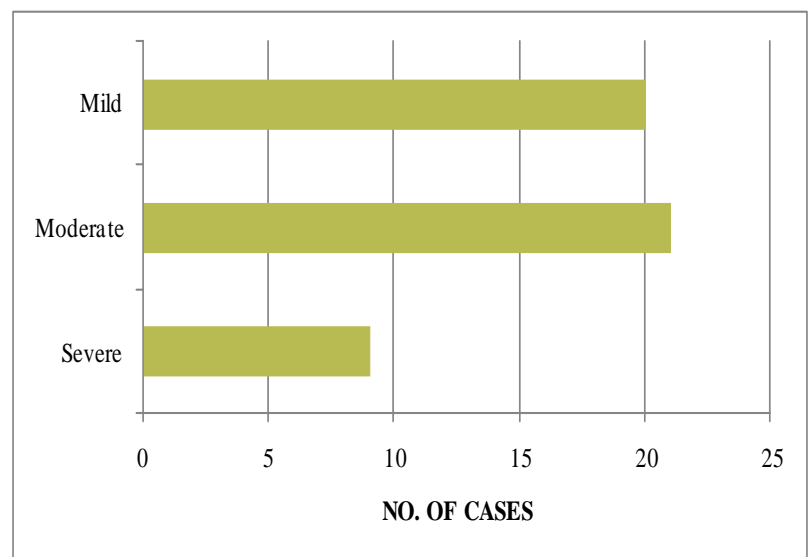

Figure 1; Grading of acute pancreatitis According to Modified CT severity index

\section{PATIENT OUTCOME PARAMETERS}

ORGAN FAILURE - 22 out of 50 patients developed organ failure. Of these 22 patients who developed end organ failure, 6 patients had mild, 7 patients had moderate and 9 patients had severe pancreatitis according to the MCTSI.

$30 \%$ of patients who had mild pancreatitis had end organ failure, whereas end organ failure is seen in $33 \%$ and $100 \%$ of patients who had moderate and severe pancreatitis respectively $(\mathrm{p}<0.002)$
SYSTEMIC INFECTION- A total of 20 (40\%) patients who had fever and leukocytosis were considered to have systemic infection. Of these 20 patients, 3 patients had mild, 9 patients had moderate and 8 patients had severe pancreatitis according to the MCTSI. $15 \%$ of patients who had mild pancreatitis had systemic infection, whereas systemic infection is seen in $42 \%$ and $88 \%$ of patients who had moderate and severe pancreatitis respectively $(\mathrm{p}=0.001)$

\section{SURGICAL INTERVENTION- Surgical} intervention was required in $4(8 \%)$ patients. Two patients who underwent laparotomy had infected pseudocyst. Two patient underwent surgical necrosectomy and subsequentlyone among them expired.

In our study, none of the patients who had mild pancreatitis according to MCTSI scoring underwent surgical intervention. Two patients (4\%) had infected pseudocyst belonged to group of moderate pancreatitis, underwent surgical intervention. Two patients who had severe pancreatitis underwent surgical intervention of surgical necrosectomy. The statistical $p$ valueis more than 0.1 which is not significant.

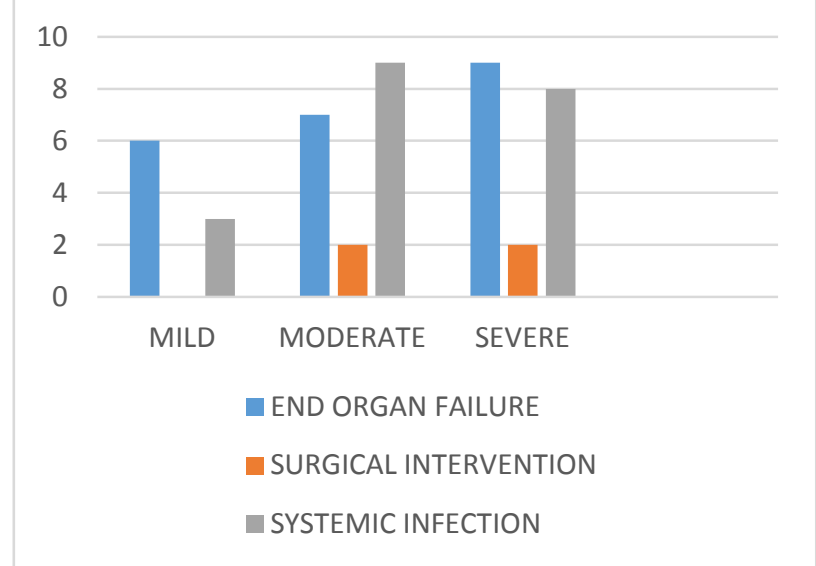

Figure 2: Correlation of Patient outcome parameters with Modified CT severity index

DURATION OF HOSPITALISATION- Duration of hospital stay in our study was ranging from 0 to 19 days with mean duration of 9 days. The mean duration of hospitalisation in mild, moderate and severe classes of acute pancreatitis according to Modified CT Severity Index was 7, 9 and 14 days respectively. The statistical $\mathrm{p}$ value is 0.02 , is significant. 


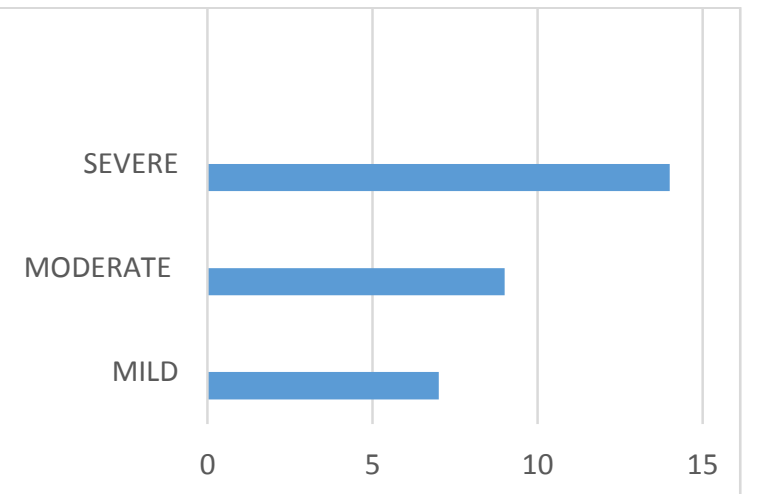

口 DURATION OF HOSPITALISATION

Figure 3 ; Correlation of Duration of hospital stay with Modified CT severity index

MORTALITY- One patient who had severe pancreatitis (score 10) based modified CT severity classification died due to shock and multiple organ failure. The same patient underwent surgical necrosectomy and died on 4th day of hospitalisation. Mortality rate in our study was $2 \%$.

\section{DISCUSSION}

In the study of 2 years (September 2014-september 2016) conducted in the department of radiodiagnosis , V S S Institute of Medical Science and Research, Burla, total 50 patients were analysed for patient outcome parameters by modified CT severity index. Mean age of presentation is 40 years in our study population. It is concurrent with study done by Thomas et al ${ }^{[1]}$, Jauregui et al ${ }^{[2]}$. The prevalence of chronic alcohol abuse and biliary stones are common in fourth and fifth decades explain the high incidence of the acute pancreatitis in this age group. Considering the clinical presentation in our study, abdominal pain was the commonest symptom as seen in all the 50 cases (100\%) followed by vomiting in 13 cases (26\%) which correlates with the study by Mortele et al ${ }^{[3]}$, Bollen et al ${ }^{[4]}$. O'Connor et al ${ }^{[5]}$, BC KOO et al ${ }^{[6]}$, and $\mathrm{BU}$ Wu et al ${ }^{[7]}$

$22(44 \%)$ out of 50 patients developed organ failure and significant correlation between severity of pancreatitis and development of end organ failure was noted using modified CT severity index but Bollen et al ${ }^{[4]}$ showed 38 (19\%) out of 196patients of organ failure. The differences observed may be due to differences in criteria for organ failure and clinically severe AP (the present study used criteria in accordance with the Marshall criteria of end organ failure ${ }^{[8]}$. Mortality in our study was $2 \%$ which correlates with Mortele et al ${ }^{[3]}$ who showed $3 \%$ mortality in a total 66 patients. Duration of hospitalisation in mild, moderate and severe variants of pancreatitis showed statistically significant correlation with modified ct severity scoring of pancreatitis. In contrary to Mortele et al [3] modified CT severity index did not show significant correlation with surgical intervention in our study that may be due to infected pseudocyst in two patients who presented with relapse of acute pancreatitis with different modified ct severity scores.

\section{LIMITATIONS}

All patients with acute attacks of pancreatitis were included in study irrespective of whether first attack or relapse of pancreatitis. Hence, difference between first attacks and relapses could not be differentiated. The sample size was inadequate to evaluate mortality and morbidity prediction based on CT criteria

\section{CONCLUSION}

To conclude with, Modified CT severity index is a good prognostic indicator of outcome of patients with acute pancreatitis. it showed significant correlation with patient outcomes especially with end organ failure, duration of hospitalization and systemic infection. No significant correlation was noted with surgical intervention.

\section{REFERENCES}

1. Thomas L Bollen, Vikesh K Singh, Rie Maurer, Kathryn Repas, Hendrik W van Es, Peter A Banks and Koenraad J Mortele. A Comparative Evaluation of Radiologic and Clinical Scoring Systems in the Early Prediction of Severity in Acute Pancreatitis. The American Journal of Gastroenterology 107, 612-619 (April 2012). 
2. Jauregui-Arrieta L, Alvarez-Lopez F, Cobian-Machuca H, Solis-Ugalde J, TorresMendoza B, Troyo-Sanroman R. Effectiveness of the modify tomographic severity index in patients with severe acutepancreatitis. Rev Gastroenterol Mex. 2008 Jul- Sep; 73(3):144-8

3. Koenraad J. Mortele, Walter Wiesner, Lisa Intriere, Shridhar Shankar, Kelly H. Zou1, Babek N. Kalantari, Alex Perez, Eric van Sonnenberg, Pablo R. Ros, Peter A. Banks, Stuart G. Silverman: A Modified CT Severity Index for Evaluating Acute Pancreatitis: Improved Correlation with Patient Outcome, AJR: 2004, November.183.

4. Thomas L. Bollen, Vikesh K. Singh, Rie Maurer, Kathryn Repas, Hendrik W. van Es, Peter A. Banks, Koenraad J. Mortele: Comparative Evaluation of the Modified CT Severity Index and CT Severity Index in Assessing Severity of Acute Pancreatitis, AJR: 2011 August, 197. Balthazar: CT diagnosis and staging of acute pancreatitis, RadiolClin. North America 27:19, 1989.

5. Owen J. O'Connor, Sebastian McWilliams, Michael M. Maher: Imaging of Acute Pancreatitis, 1Department of Radiology, Cork University Hospital, University College Cork, Wilton, Cork, Ireland. AJR: 2011 August, 197

6. B C Koo, A Chinogureyi, and A S Shaw: Imaging acute pancreatitis, The British Journal of Radiology. 2010;83:104-112.

7. B U Wu, R S Johannes, X Sun, Y Tabak, D L Conwell, P A Banks The early prediction of mortality in acute pancreatitis: a large population-based study Gut. 2008;57:16981703. doi:10.1136/gut.2008.152702

8. MarshallJC,CookDJ,ChristouNV,etal:Multip leorgan dysfunction score: An reliable descriptor of a complex clinical outcome. CritCareMed 1995;23:1638-52.

9. Balthazar E J, Freeny PC, Van Sonneberg E Imaging and intervention in acute pancreatitis. Radiology. 1994;193;23: 898905.

10. John Treacy, Anthony Williams, Renz Bais, Krysten Willson, Christopher Worthley, John Reece, Justin Bessell, David Thomas. Evaluation of amylase and lipase in the diagnosis of acute pancreatitis. ANZ Journal of Surgery, 2001, Volume 71, Issue 10,577-58.

11. Bradley 3rd EL. A clinically based classification system for acute pancreatitis. Arch Surg 1993; 128:586. 